M.V. Yarmolenko

\title{
Copper and Aluminum Electric Corrosion Investigation and Intermetallics Disappearance in Cu-Al System Analysis
}

\author{
Kyiv National University of Technologies and Design, Faculty of Market, Information and Innovation Technologies, \\ Cherkasy, Ukraine, yarmolenko.mv@knutd.edu.ua
}

\begin{abstract}
Copper and aluminum electric corrosion is investigated experimentally. It is founded that copper corrosion is higher than aluminum corrosion. Intermetallics disappearance rate in $\mathrm{Cu}-\mathrm{Al}$ system is analyzing theoretically. Literature experimental data are used for analysis.
\end{abstract}

Key words: copper, aluminum, electrolysis, diffusion, intermetallics, phases formation kinetics.

Received 22 March 2020; Accepted 15 June 2020.

\section{Introduction}

Mutual diffusion in $\beta$-phase $\left(C_{A l} \approx 0.24 \pm 0.02\right)$ of $\mathrm{Cu}-\mathrm{Al}$ system was investigated at temperatures from $700{ }^{\circ} \mathrm{C}$ to $840{ }^{\circ} \mathrm{C}$ ( $\beta$-phase can exist at temperatures that are higher than $565^{\circ} \mathrm{C}$ ) [1]. The phase formation kinetics between $\alpha$-phase $\left(C_{A l} \approx 0.15\right)$ and $\gamma$-phase $\left(C_{A l} \approx 0.31\right)$ was analyzed, and $\beta$-phase formation kinetics between pure copper $(99.99 \% \mathrm{Cu})$ and $\gamma$-phase $\left(C_{A l} \approx 0.31\right)$ was analyzed too. The phase formation parabolic law was observed. The pre-exponential factor and mutual diffusion activation energy for diffusion in $\beta$-phase were calculated: $D_{0} \approx 1.3 \cdot 10^{-5} \mathrm{~m}^{2} / \mathrm{s}, Q \approx 27 \pm(3 \div 5) \mathrm{kcal} / \mathrm{mol}$. The pre-exponential factor and $\beta$-phase formation activation energy were calculated: $D_{0} \approx 10^{-2} \div 10^{-1} \mathrm{~m}^{2} / \mathrm{s}$, $Q \approx 40 \pm 3 \mathrm{kcal} / \mathrm{mol}$. It was proved experimentally that mutual diffusion penetrability of $\beta$-phase does not depend on annealing time and initial composition. It was proved experimentally [2] that copper corrosion rate is higher than gold corrosion rate, but intermetallics formation rate in $\mathrm{Au}-\mathrm{Al}$ system is much more higher than intermetallics formation rate in $\mathrm{Cu}-\mathrm{Al}$ system, so it is possible to use $\mathrm{Cu}$ instead of $\mathrm{Au}$ for wire bonding in microelectronics packaging, because thin $\mathrm{Al}$ pad $(1.2 \mu \mathrm{m}$ thickness) can prevent gold and copper corrosion, and $\mathrm{Cu}$ has higher electric conductivity, higher thermal conduction, and lower material cost than $\mathrm{Au}$. Theoretical method was proposed to describe intermetallics disappearance rate in double multiphase systems [3].
Another theoretical method was described to calculate intermetallics formation rate in double multiphase systems [4]. Three phases growth kinetics ( $\varepsilon$-brass, $\mathrm{CuZn}_{5}, C_{1} \approx 0.83 ; \gamma$-brass, $\mathrm{Cu}_{5} \mathrm{Zn}_{8}, C_{2} \approx 0.62$; and $\beta$ brass, $\mathrm{CuZn}, C_{3} \approx 0.5$ ) was analyzed theoretically and experimentally at $230{ }^{\circ} \mathrm{C}, 340{ }^{\circ} \mathrm{C}$, and $400{ }^{\circ} \mathrm{C}$ in planar and cylindrical samples $[5,6,13,14]$, and theoretically in spherical samples [15].

Electric current can destruct wire bonding in microelectronics packaging, so we planned to investigate copper and aluminum electric corrosion. Direct current can dissolve metal anode into electrolyte, and we planned to do experiments under the same conditions: initial radii of $\mathrm{Al}$ and $\mathrm{Cu}$ anodes should be equal; electrolyte concentration should be the same; anodes lengths immersed into electrolyte should be approximately equal; graphite cathodes should be the same; direct electric current value should be practically the same.

\section{Experimental results of copper and aluminum electric corrosion investigation}

Cylindrical anodes $(99.99 \% \mathrm{Cu}$ and $99.99 \% \mathrm{Al})$ were used for copper and aluminum electric corrosion investigation. Sodium chloride $(\mathrm{NaCl})$ solution was used as electrolyte (Fig. 1). Direct electric current and anodes 


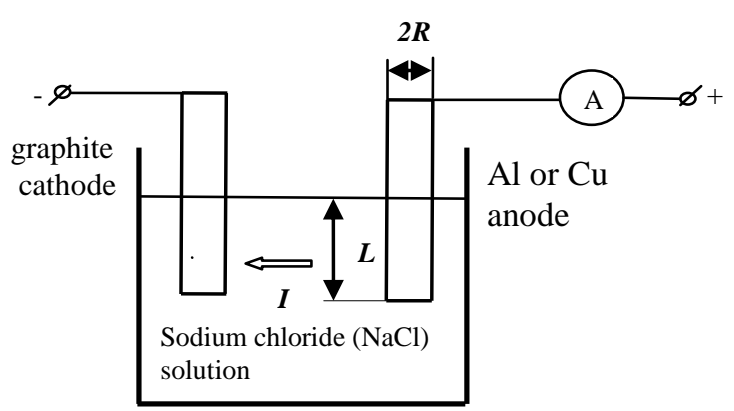

Fig. 1. Scheme of experimental equipment.

mass decreasing were measured. First of all, we need to be assured that ions $\mathrm{Cu}^{+}\left(\right.$or $\left.\mathrm{Cu}^{2+}\right)$ and $\mathrm{Al}^{3+}$ were present in $\mathrm{NaCl}$ solution. Rate of anode dissolving into electrolyte can be calculated using Faraday's law of electrolysis:

$$
\frac{d m}{d t}=\frac{M I}{z F}, d m=\rho \cdot L \cdot \pi \cdot d\left(R^{2}(t)\right),
$$

where $m$ is anode mass dissolved into electrolyte, $t$ is time of experiment, $M$ is molar mass, $I$ is direct electric current value, $F$ is the Faraday constant $(F=$ 96485.33289(59) $\mathrm{C} \mathrm{mol}^{-1}$ ), $z$ is charge of ions, $R$ is anode radius, $L$ is anode length immersed into electrolyte. Electric current value did not change, so one can calculate:

$$
z=\frac{M I t}{F \pi \rho L\left(R^{2}(t=0)-R^{2}(t)\right)},
$$

where $\rho$ is anode density. Charges of copper and aluminum ions were calculated:

$$
\begin{aligned}
& z_{C u}=\frac{63.55 \cdot 10^{-3} \mathrm{~kg} / \mathrm{mol} \cdot 2.8 \mathrm{~A} \cdot 1.2 \cdot 10^{3} \mathrm{~s}}{F \cdot \pi \cdot 8.9 \cdot 10^{3} \mathrm{~kg} / \mathrm{m}^{3} L_{C u} \cdot\left(R_{C u}^{2}(t=0)-R_{C u}^{2}\left(t_{4}\right)\right)} \approx 0.995 \approx 1,
\end{aligned}
$$

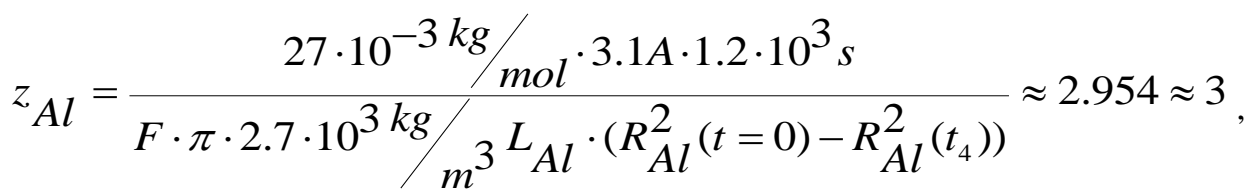

where $\quad L_{C u} \approx L_{A l}: \quad L_{C u}=5 \cdot 10^{-2} \mathrm{~m}, \quad L_{A l}=4.5 \cdot 10^{-2} \mathrm{~m}$; $R_{O C u}=R_{O A l}=2.8 \mathrm{~m} ; I_{A l} \approx I_{C u}: I_{A l}=3.1 \mathrm{~A}, I_{C u}=2.8 \mathrm{~A}$, so copper dissolved into $\mathrm{NaCl}$ solution as $\mathrm{Cu}^{+}$ions, and aluminum dissolved into $\mathrm{NaCl}$ solution as $\mathrm{Al}^{3+}$ ions. Anodes radii decreasing kinetics is shown on Fig.2. Experiments were carried during $t_{1}=5 \mathrm{~min}, t_{2}=10 \mathrm{~min}$, $t_{3}=15 \mathrm{~min}$, and $t_{4}=20 \mathrm{~min}$. Experimental results are as follows: $\quad R_{l \mathrm{Cu}}=2.74 \mathrm{~mm}, \quad R_{2 \mathrm{Cu}}=2.67 \mathrm{~mm}$, $R_{3 \mathrm{Cu}}=2.59 \mathrm{~mm}, \quad R_{4 \mathrm{Cu}}=2.5 \mathrm{~mm} ; \quad R_{l \mathrm{Al}}=2.77 \mathrm{~mm}$, $R_{2 A l}=2.73 \mathrm{~mm}, \quad R_{3 A l}=2.68 \mathrm{~mm}, \quad R_{4 A l}=2.62 \mathrm{~mm}$. Measurement precision was $0.01 \mathrm{~mm}$.

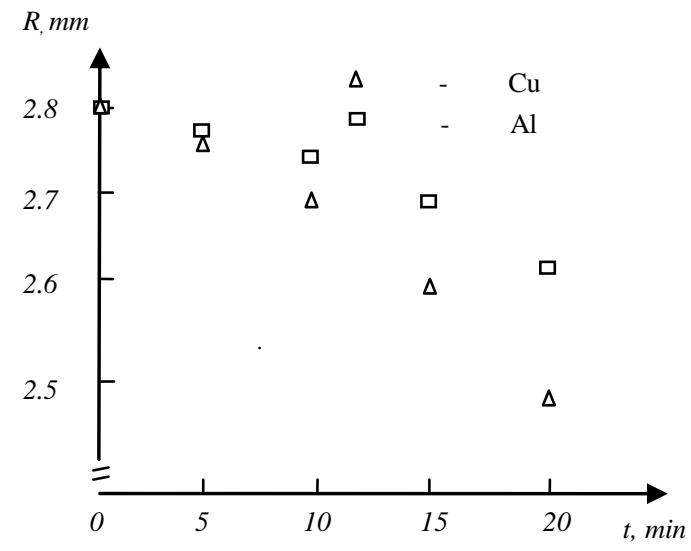

Fig. 2. $\mathrm{Al}$ and $\mathrm{Cu}$ anodes radii decreasing kinetics.
Chemical reactions took place near positive electrode (anode):

$$
\begin{gathered}
C u^{+}+C l^{-}=C u C l \downarrow \\
A l^{3+}+3 C l^{-}=A l C l_{3} \downarrow \\
C u^{+}-e^{-}=C u^{2+}, \\
C u^{2+}+2 C l^{-}=C u C l_{2} \downarrow \\
C l^{-}-e^{-}=C l^{0}, C l^{0}+C l^{0}=C l_{2} \uparrow .
\end{gathered}
$$

Chlorine gas was formed near anode.

Chemical reactions took place near negative electrode (cathode):

$$
\begin{gathered}
\mathrm{Na}^{+}-e^{-}=\mathrm{Na}^{\mathrm{O}} \\
2 \mathrm{Na}+2 \mathrm{H}_{2} \mathrm{O}=2 \mathrm{NaOH}+\mathrm{H}_{2} \uparrow .
\end{gathered}
$$

Hydrogen gas was formed near anode.

Anodes radii decreasing rate constants can be calculated as average value of four experiments:

$$
k_{C u}=\frac{4 R_{0}^{2}-\sum_{i=1}^{4} R_{i}^{2}}{\sum_{i=1}^{4} t_{i}} \approx 1.25 \cdot 10^{-9} \mathrm{~m}^{2} / \mathrm{s},
$$




$$
\begin{gathered}
k_{A l}=\frac{4 R_{0}^{2}-\sum_{i=1}^{4} R_{i}^{2}}{\sum_{i=1}^{4} t_{i}} \approx 7.29 \cdot 10^{-10} \mathrm{~m}^{2} / \mathrm{s}, \\
{ }^{k} \mathrm{Cu}
\end{gathered}
$$

so copper electric corrosion is much more higher than aluminum electric corrosion, despite of $I_{A l} \geq I_{C u}: I_{A l} \approx$ $1.1 I_{C u}$. It is need to point out that $k_{C u}$ and $k_{A l}$ have dimensionalities as diffusion coefficient because the anodes had cylindrical shape.

\section{Analysis of intermetallics disappearance rate in $\mathrm{Al}-\mathrm{Cu}$ system}

There are three phases in $\mathrm{Cu}-\mathrm{Al}$ system at temperatures from $175{ }^{\circ} \mathrm{C}$ to $225{ }^{\circ} \mathrm{C}$ [2]: $\mathrm{CuAl}_{2}\left(C_{l}=\right.$ $2 / 3 \approx 0.67), \mathrm{CuAl}\left(C_{2}=1 / 2=0.5\right)$, and $\gamma$-phase $\mathrm{Cu}_{9} \mathrm{Al}_{4}$ $\left(C_{3}=4 / 13 \approx 0.31\right), C=C_{A l}$. Phases 1,2 , and 3 formation rates between thin $\mathrm{Al}$ layer and $\mathrm{Cu}$ can be calculated, using so-called "constant flux method" (K.P. Gurov and A.M. Gusaks' method) [4]:

$$
\begin{array}{r}
\frac{d X_{1}}{d t} \approx \frac{1-C_{2}}{\left(1-C_{1}\right)\left(C_{1}-C_{2}\right)} \frac{D_{1}}{X_{1}}-\frac{1}{C_{1}-C_{2}} \frac{D_{2}}{X_{2}}=\frac{K_{1}}{2 X_{1}}, \quad X_{1}^{2}=K_{1} t, \\
\frac{d X_{2}}{d t} \approx \frac{C_{1}-C_{3}}{\left(C_{1}-C_{2}\right)\left(C_{2}-C_{3}\right)} \frac{D_{2}}{X_{2}}-\frac{1}{C_{1}-C_{2}} \frac{D_{1}}{X_{1}}-\frac{1}{C_{2}-C_{3}} \frac{D_{3}}{X_{3}}=\frac{K_{2}}{2 X_{2}}, \\
X_{2}^{2}=K_{2} t, \frac{d X_{3}}{d t} \approx \frac{C_{2}}{C_{3}\left(C_{2}-C_{3}\right)} \frac{D_{3}}{X_{3}}-\frac{1}{C_{2}-C_{3}} \frac{D_{2}}{X_{2}}=\frac{K_{3}}{2 X_{3}}, X_{3}^{2}=K_{3} t, \\
\left(X_{1}+X_{2}+X_{3}\right)^{2}=X^{2}=K_{123} t, \quad K_{123}=\left(\sqrt{K_{1}}+\sqrt{K_{2}}+\sqrt{K_{3}}\right)^{2},
\end{array}
$$

where $D_{i}(i=1 ; 2 ; 3)$ is mutual diffusion penetrability of phase $i\left(D_{i}=D_{i}{ }^{*} \cdot \Delta C_{i}\right), D_{i}{ }^{*}$ is mutual diffusion coefficient in phase $i, \Delta C_{i}$ is narrow concentration range of homogeneity. $K_{1}$ depends on $D_{1}$ and $D_{2}, K_{2}$ depends on $D_{1}, D_{2}$ and $D_{3}$, and $K_{3}$ depends on $D_{3}$ and $D_{2}$. Aluminum disappearance time, $t_{0}$, can be estimated by the following way. Mass conservation law gives (Fig. 3):

and

$$
\begin{aligned}
& X_{A l}(t=0) \cdot 1=C_{1} X_{1}\left(t_{0}\right)+C_{2} X_{2}\left(t_{0}\right)+C_{3} X_{3}\left(t_{0}\right)= \\
& =C_{1}\left(\sqrt{K_{1}}+\frac{C_{2}}{C_{1}} \sqrt{K_{2}}+\frac{C_{3}}{C_{1}} \sqrt{K_{3}}\right) \sqrt{t_{0}} \approx C_{1} \sqrt{K_{123}} \sqrt{t_{0}} \\
& t_{0}=\frac{X_{A l}^{2}}{\left(C_{1} \sqrt{K_{1}}+C_{2} \sqrt{K_{2}}+C_{3} \sqrt{K_{3}}\right)^{2}} \approx \frac{X_{A l}^{2}}{C_{1}^{2} K_{123}} .
\end{aligned}
$$

Two phases (phase 2 and phase 3) are formed between phase 1 and copper after disappearance of aluminum:

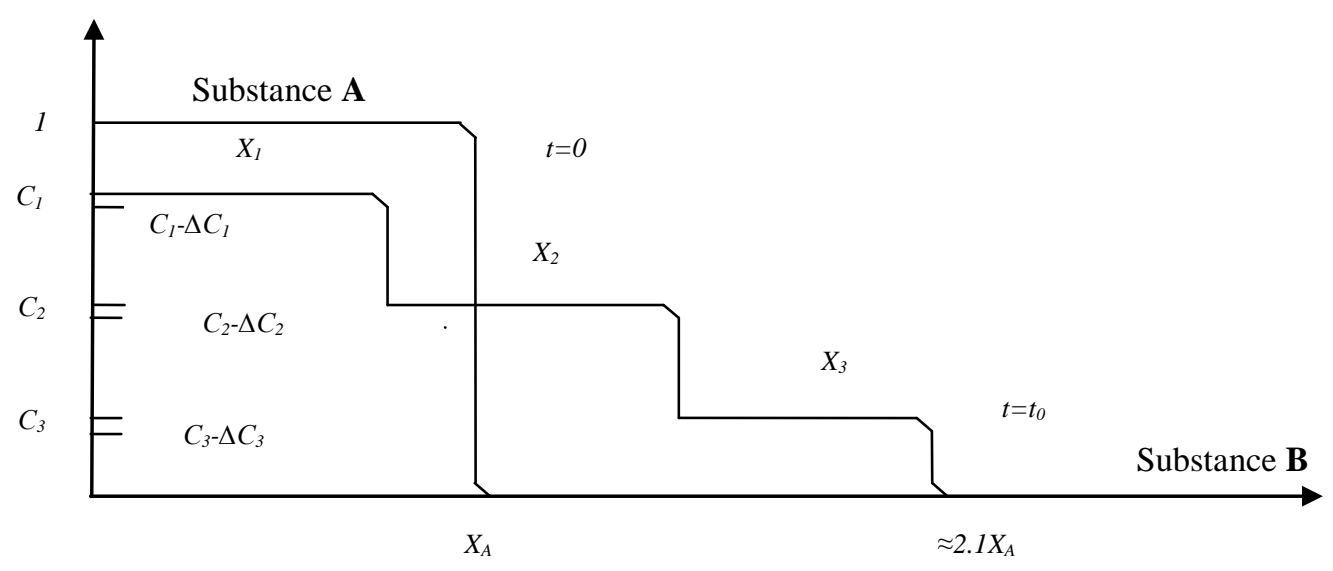

Fig. 3. Concentration profile change during isothermal annealing from $t=0$ to $t=t_{0}$. 


$$
\begin{aligned}
\frac{d X_{2}}{d t} & \approx \frac{C_{1}-C_{3}}{\left(C_{1}-C_{2}\right)\left(C_{2}-C_{3}\right)} \frac{D_{2}}{X_{2}}-\frac{1}{C_{2}-C_{3}} \frac{D_{3}}{X_{3}}=\frac{K_{2}}{2 X_{2}}, \quad X_{2}^{2}=K_{2} t, \\
\frac{d X_{3}}{d t} & \approx \frac{C_{2}}{C_{3}\left(C_{2}-C_{3}\right)} \frac{D_{3}}{X_{3}}-\frac{1}{C_{2}-C_{3}} \frac{D_{2}}{X_{2}}=\frac{K_{3}}{2 X_{3}}, \quad X_{3}^{2}=K_{3} t, \\
\left(X_{2}+X_{3}\right)^{2} & =X^{2}=K_{23} t, K_{23}=\left(\sqrt{K_{2}}+\sqrt{K_{3}}\right)^{2} \approx \frac{4}{9} K_{123}, \text { if } K_{1} \approx K_{2} \approx K_{3} .
\end{aligned}
$$

Mass conservation law gives (Fig. 4):

$$
\begin{gathered}
X_{A l}(t=0) \cdot 1=C_{2} X_{2}\left(t_{1}\right)+C_{3} X_{3}\left(t_{1}\right)=\sqrt{t_{1}} C_{2}\left(\sqrt{K_{2}}+\frac{C_{3}}{C_{2}} \sqrt{K_{3}}\right), \\
t_{1} \approx \frac{X_{A l}^{2}}{C_{2}^{2} K_{23}} \approx \frac{9 X_{A l}^{2}}{4 C_{2}^{2} K_{123}} .
\end{gathered}
$$

and

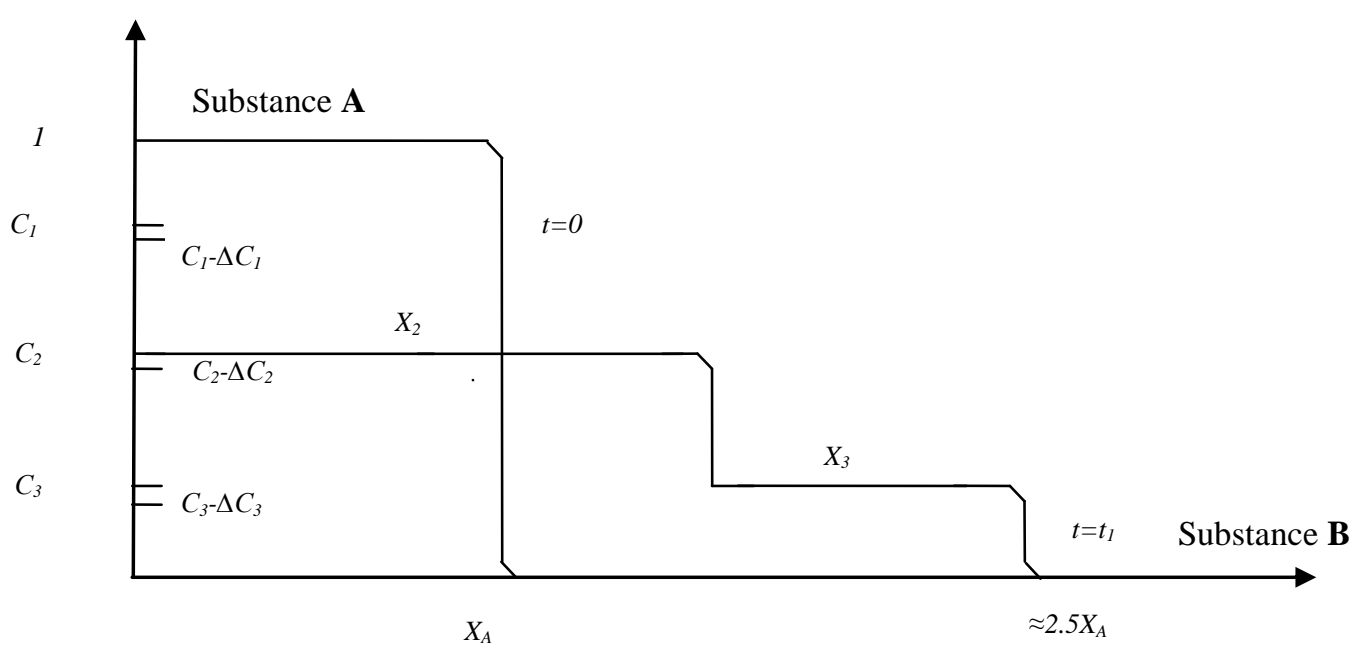

Fig. 4. Concentration profile change during isothermal annealing from $t=0$ to $t=t_{l}$.

Phase 3 is formed between phase 2 and copper after disappearance of aluminum and phase 1 :

$$
\frac{d X_{3}}{d t} \approx \frac{C_{2}}{C_{3}\left(C_{2}-C_{3}\right)} \frac{D_{3}}{X_{3}}=\frac{K_{3}}{2 X_{3}}, \quad X_{3}^{2}=K_{3} t \approx \frac{1}{9} K_{123} t .
$$

Mass conservation law gives (Fig. 5):

$$
\begin{gathered}
X_{A l}(t=0) \cdot 1=C_{3} X_{3}\left(t_{2}\right)=\sqrt{t_{2}} C_{3} \sqrt{K_{3}}, \text { and } \\
t_{2} \approx \frac{X_{A l}^{2}}{C_{3}^{2} K_{3}} \approx \frac{9 X_{A l}^{2}}{C_{3}^{2} K_{123}} .
\end{gathered}
$$




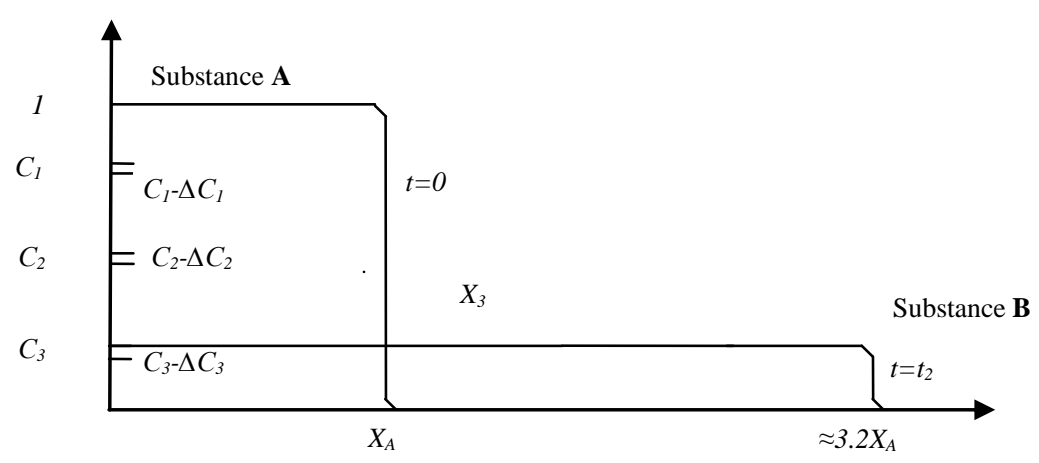

Fig. 5. Concentration profile change during isothermal annealing from $t=0$ to $t=t_{2}$.

We can analyze described experimental results [2]. The $\mathrm{Au}$ and $\mathrm{Cu}$ wires were bonded to the $\mathrm{Al} / 0.5 \% \mathrm{Cu}$ pad of $X_{A l}=1.2 \mu \mathrm{m}$ thickness using the ASM Eagle $60 \mathrm{AP}$ wire bonder. The $\mathrm{Au}$ and $\mathrm{Cu}$ wires were annealing at temperatures $T_{1}=175{ }^{\circ} \mathrm{C}, T_{2}=200{ }^{\circ} \mathrm{C}$, and $T_{3}=225{ }^{\circ} \mathrm{C}$ during $120 \mathrm{~h}, 240 \mathrm{~h}, 360 \mathrm{~h}$ and $480 \mathrm{~h}$. Phase layers thicknesses for $\mathrm{Cu} / \mathrm{Al}$ were calculated:

$$
X^{2}=K_{123} t+K_{01}=3.52 \cdot 10^{-4 \mu m^{2}} / s \cdot e^{(-3064.5 / T)} t+0.44 \mu m^{2}
$$

where $K_{01}$ is constant related to initial IMC thickness. General reaction rates of IMC formation were calculated:

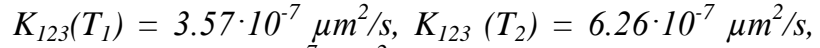
$K_{123}\left(T_{3}\right)=7.15 \cdot 10^{-7} \mu \mathrm{m}^{2} / \mathrm{s}$. The pre-exponential factor and IMC formation activation energy were calculated: $K_{0} \approx 3.52 \cdot 10^{-4} \mu \mathrm{m}^{2} / \mathrm{s}, Q \approx 6.1 \mathrm{kcal} / \mathrm{mol}$. We can calculate aluminum disappearance time for different temperatures using Equation (11):

$$
\begin{gathered}
t_{0}\left(T_{1}\right) \approx 2520 \mathrm{~h}, \mathrm{t}_{0}\left(T_{2}\right) \approx 1440 \mathrm{~h}, \\
t_{0}\left(T_{3}\right) \approx 1260 \mathrm{~h}, \mathrm{t} \approx \frac{480 \mathrm{~h}}{1260 \mathrm{~h}} \mathrm{t}_{0} \approx 0.4 \mathrm{t}_{0},
\end{gathered}
$$

so all three phases are formed in diffusion zone (Fig. 6):

$$
X_{A}\left(t=0.4 t_{0}\right) \approx C_{1} \sqrt{K_{123} t_{0}} \sqrt{1-0.4} \approx 0.77 X_{A}(t=0)
$$

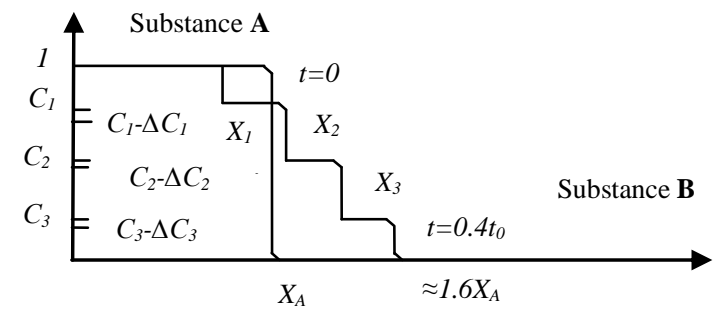

Fig. 6. Concentration profile change during isothermal annealing in $\mathrm{Al}-\mathrm{Cu}$ system from $t=0$ to $t=0.4 t_{0}$.

Phase 1 disappearance time at temperature $T_{3}$ can be calculated using Equation (13):

$$
t_{1}\left(T_{3}\right) \approx 5035 h \approx 7 \text { months }
$$

Phase 2 disappearance time can be calculated using Equation (15):

$$
t_{2}\left(T_{3}\right) \approx 52390 h \approx 6 \text { years } .
$$

By the way, assumption $K_{l} \approx K_{2} \approx K_{3}$ is incorrect [5, $6,13]$, so obtained estimations should be less in several times. Equations (11), (13), and (15) correctly describe obtained experimental results $[5,6]$.

\section{Conclusions}

Copper electric corrosion is much more higher than aluminum electric corrosion, ${ }^{k} \mathrm{Cu} \approx 1,72 k_{A l}$, so thin $\mathrm{Al}$ pad can prevent copper electric corrosion. Anodes radii decreasing rate constants, $k_{C u}$ and $k_{A l}$, have dimensionalities as diffusion coefficient, because the anodes had cylindrical shape.

Thin metal covering disappearance time, $t_{0}$, can be estimated by the following way:

$$
t_{0}=\frac{X_{A}^{2}}{\left(C_{1} \sqrt{K_{1}}+C_{2} \sqrt{K_{2}}+C_{3} \sqrt{K_{3}}\right)^{2}} \approx \frac{X_{A}^{2}}{C_{1}^{2} K_{123}},
$$

where $X_{A}$ is initial metal covering thickness, $C_{I}$ is concentration in phase $1, K_{123}$ is general reaction rates of IMC formation, $C_{1}>C_{2}>C_{3} ; K_{1}, K_{2}$, and $K_{3}$ are phases 1 , 2 , and 3 formation rates.

Phase 1 disappearance time, $t_{1}$, can be calculated:

$$
t_{1} \approx \frac{X_{A}^{2}}{C_{2}^{2} K_{23}} \approx \frac{9 X_{A}^{2}}{4 C_{2}^{2} K_{123}} .
$$

Phase 2 disappearance time, $t_{2}$, can be calculated:

$$
t_{2} \approx \frac{X_{A}^{2}}{C_{3}^{2} K_{3}} \approx \frac{9 X_{A}^{2}}{C_{3}^{2} K_{123}} .
$$

It is need to point out that K.P. Gurov and A.M.Gusaks' method can be applied to describe phase formation rate along GB with outflow in volume [7, 8, 9] 
(J.C.Fisher's model [10]) and along dislocation pipe with outflow in volume [11] (A.D. LeClaire and A. Rabinovichs' model [12]).
Yarmolenko M.V. - PhD, Associate Professor, Head of the Chair of Information and Computer Technologies and Fundamental Disciplines.

[1] V.N. Pimenov, K.A. Akkushkarova, and Yu.E. Ugaste, Fizika metallov i metalloved. 39(4) 821 (1975).

[2] C.S. Goh, W.L.E. Chong, T.K. Lee, and C. Breach, Crystals 3, No.3, 391 (2013) (https://doi.org/10.3390/cryst3030391).

[3] V.I. Neverov, Yu.E. Ugaste, Fizika i Khimia obrabotki materialov 5, 113 (1992).

[4] K.P. Gurov, A.M. Gusak, and M.V. Yarmolenko, Metallofizika 10(3) $91 \quad$ (1988) (https://mfint.imp.kiev.ua/ru/toc/v10/i05.html).

[5] V.V. Bogdanov, A.M. Gusak, L.N. Paritskaya, and M.V. Yarmolenko, Metallofizika 12(3) 60 (1990).

[6] V.V. Bogdanov, L.N. Paritskaya, and M.V. Yarmolenko, Metallofizika 12(5) 98 (1990).

[7] M.V. Yarmolenko, A.M. Gusak, and K.P. Gurov, Journal of Engineering Physics and Thermophysics 65, 876 (1993) (https://doi.org/10.1007/BF00862930).

[8] M.V. Yarmolenko, Defect and Diffusion Forum 143-147, 1567 (1997) (https://doi.org/10.4028/www.scientific.net/DDF.143-147.1567).

[9] M.V. Yarmolenko, Metallofiz. Noveishie Tekhnol. 40(9), 1201 (2018) (DOI: 10.15407/mfint.40.09.1201).

[10] J.C. Fisher, J. Appl. Phys. 22(1), 1699825 (1951) (https://doi.org/10.1063/1.1699825).

[11] M.V. Yarmolenko, AIP Advances 8, 095202 (2018) (https://doi.org/10.1063/1.5041728).

[12] A.D. Le Claire and A. Rabinovich, J. Phys. C: Solid State Phys. 15, 5727 (1982) (https://iopscience.iop.org/article/10.1088/0022-3719/15/16/007).

[13] A.M. Gusak and M.V. Yarmolenko, J. Appl. Phys. 73(10), 4881 (1993) (https://doi.org/10.1063/1.353805).

[14] M.V. Yarmolenko, Deviation from Parabolic Growth of Phase Layers in Cylindrical and Spherical Samples: Curvature and Internal Stress Influence in Proceedings of PTM-1994, W.C. Johnson, J.M. Howe, D.E. Laughlin, and W.A. Soffa (The Minerals, Metals \& Materials Society, Pennsylvania, 1994), p.1177 (https://publons.com/publon/3639123/).

[15] M.V. Yarmolenko, Izvestiya AN SSSR. Metally 3, 187 (1990) (https://publons.com/publon/2915981/).

\author{
М.В. Ярмоленко
}

\title{
Дослідження електричної корозії міді і алюмінію та аналіз кінетики зникнення інтерметалідів у системі $\mathrm{Cu}-\mathrm{Al}$
}

Київський національний університет технології і дизайну, Черкаси, Украӥна, yarmolenko.mv@knиtd.edu.иа

Експериментально досліджено електричну корозію міді та алюмінію. Отримано такий результат: електрична корозія міді значно швидша, ніж електрична корозія алюмінію, тому тонке алюмінієве покриття товщиною близько 1 мікрометра на мідних дротинках може уповільнити корозію міді у приладах мікроелектроніки. Теоретично проаналізовано процес зникнення інтерметалідів у системі $\mathrm{Cu}-\mathrm{Al}$. Для аналізу були використані літературні експериментальні дані.

Ключові слова: мідь; алюміній; електроліз; дифузія; інтерметаліди; кінетика утворення фаз. 